\title{
miR-203 contributes to IL-17-induced VEGF secretion by targeting SOCS3 in keratinocytes
}

\author{
YUANYUAN XU ${ }^{1}$, YONGZHI JI ${ }^{1,2}$, XIAOOU LAN ${ }^{1}$, XINGHUA GAO $^{1}$, HONG-DUO CHEN $^{1}$ and LONG GENG ${ }^{1}$ \\ ${ }^{1}$ Department of Dermatology, The First Hospital of China Medical University, Heping, Shenyang, Liaoning 110001; \\ ${ }^{2}$ Department of Dermatology, The Second Hospital of Jilin University, Changchun, Jilin 130041, P.R. China
}

Received May 16, 2016; Accepted June 9, 2017

DOI: $10.3892 / \mathrm{mmr} .2017 .7759$

\begin{abstract}
Interleukin (IL)-17 signaling serves an important role in the development and pathogenesis of psoriasis; a chronic skin disease characterized by increased dermal vascularity and the hyperproliferation of keratinocytes. microRNA (miR)-203 is preferentially expressed in the skin and is an important regulator of keratinocyte differentiation. miR-203 has been implicated in a number of skin diseases, including psoriasis. However, the role of miR-203 in IL-17-induced vascular endothelial growth factor (VEGF) secretion has yet to be elucidated. The present study demonstrated that miR-203 expression was upregulated in the ears of IL-17-stimulated mice and IL-17-treated HaCaT cells. In addition, the IL-17-induced increase in miR-203 expression activated the Janus kinase/signal transducer and activator of transcription signaling pathway and promoted VEGF secretion in HaCaT cells. Furthermore, miR-203 was observed to bind to the 3'-untranslated region of suppressor of cytokine signaling 3 (SOCS3) and inhibited SOCS3 expression. The results suggest that miR-203 expression may be upregulated by IL-17 stimulation, and miR-203 is a positive regulator of IL-17-induced VEGF secretion. The present study may support potential therapeutic strategies for the treatment of psoriasis.
\end{abstract}

\section{Introduction}

Psoriasis is a complex skin disease involving reciprocity between immune cells and keratinocytes, and is characterized by the infiltration of multiple inflammatory cells, upregulation dermal vascularity and keratinocyte proliferation (1-4). Angiogenesis commences with early psoriatic alterations and disappears when the disease is cured. A number of pro-angiogenic mediators, including vascular

Correspondence to: Dr Long Geng, Department of Dermatology, The First Hospital of China Medical University, 155 Nanjing Street, Heping, Shenyang, Liaoning 110001, P.R. China

E-mail: genglong13@163.com

Key words: microRNA-203, interleukin-17, vascular endothelial growth factor endothelial growth factor (VEGF), tumor necrosis factor, hypoxia-inducible factor, interleukin (IL)-8, angiopoietins and IL-17, are upregulated during the development of psoriasis (5-8).

Initially, psoriasis was considered to be a T-helper (Th)1-mediated skin disease (9). However, a number of subsequent studies later demonstrated that Th17 cells, which are activated upon exposure to IL-23, IL-6 and transforming growth factor- $\beta$ and produce IL-17, IL-21 and IL-22 cytokines, serves a key role in the pathogenesis of psoriasis (9-12). IL-17 is one of the most potent proinflammatory cytokines, and is secreted by Th17 cells (9). A previous study indicated that IL-17 may induce keratinocytes to produce VEGF, which is an important angiogenic mediator (13). Therefore, elucidation of the mechanisms by which IL-17 signaling in keratinocytes is regulated may facilitate the development of novel treatments for Th17-mediated angiogenesis.

The Janus kinase/signal transducer and activator of transcription signaling (JAK/STAT) signaling pathway controls a number of important biological responses, including cellular differentiation, immune functions, hematopoiesis and cellular growth, and it is initiated when a receptor is bound by its corresponding cytokine and subsequently transmits signals from the cell surface membrane to the target genes (14). A previous study confirmed that IL-17 induced upregulation of VEGF expression via activation of the JAK2/STAT3 signaling pathway (15). However, the precise mechanisms involved required further investigation. An increasing number of studies have indicated that microRNAs (miRNAs/miRs) may beinvolved inthepathogenesisofpsoriasis, and miRNAs associated with psoriasis have been identified by comparing their expression in normal and psoriatic skin samples $(16,17)$. However, whether specific miRNAs influence the process of IL-17-induced VEGF expression remains unclear.

miR-203 is preferentially expressed in the skin and is an important regulator of keratinocyte differentiation. miR-203 has been implicated in a number of skin diseases, particularly psoriasis (18). However, the role of miR-203 in IL-17-induced VEGF secretion has yet to be elucidated. The aim of the present study was to investigate the role of miR-203 in IL-17-induced VEGF secretion to further elucidate the mechanism of miR-203 in psoriasis. 


\section{Materials and methods}

Animals and stimulation with IL-17. A total of $16 \mathrm{BALB} / \mathrm{c}$ mice (age, 6-8 weeks; weight, 18-22 g) were obtained from the Center of Experimental Animals of China Medical University (Shenyang, China). The mice were maintained in cages (4 mice/cage) under controlled conditions (temperature, $20-25^{\circ} \mathrm{C}$; humidity $40-70 \%$ ) with daily 12 -h light/dark cycles. Mice were provided with food and water ad libitum. All animal experiments were approved by the Institutional Animal Ethics Committee of China Medical University, and were performed in accordance with the Animal Care Guidelines for Experimental Animals (19). Mice were divided into the IL-17 stimulated group and control group ( $\mathrm{n}=8 \mathrm{mice} / \mathrm{group})$, then the mice were injected intradermally into each ear with $30 \mu \mathrm{l}$ PBS, either alone or with $3 \mu \mathrm{g}$ recombinant mouse IL-17A (Sigma-Aldrich; Merck KGaA, Darmstadt, Germany) using a 30-gauge needle once a day for two consecutive days.

Immunohistochemical staining. Following 1 week, all mice were sacrificed and mouse ear tissues were fixed in $4 \%$ paraformaldehyde for $48 \mathrm{~h}$ at $4^{\circ} \mathrm{C}$, and embedded in paraffin wax. Sections were cut at $4 \mu \mathrm{m}$ and mounted onto slides. The tissue sections were dewaxed in xylene, re-hydrated using a descending ethanol series, and subjected to antigen retrieval in $0.01 \mathrm{M}$ citrate buffer. Following inhibition of endogenous peroxidase by incubating samples with $3 \% \mathrm{H}_{2} \mathrm{O}_{2}$ at $37^{\circ} \mathrm{C}$ for $30 \mathrm{~min}$, the sections were subsequently incubated with anti-VEGF (cat no. sc-80442; 1:200 dilution) and cluster of differentiation (CD)34 monoclonal antibodies (cat no. sc-74499; 1:200 dilution) (both from Santa Cruz Biotechnology, Inc., Dallas, TX, USA) overnight at $4^{\circ} \mathrm{C}$. Following washing with PBS, tissue sections were incubated with a horseradish peroxidase-conjugated goat anti-mouse IgG1 secary antibody (cat no. ab97240; 1:1,000 dilution; Abcam, Cambridge, MA, USA) at $37^{\circ} \mathrm{C}$ for $30 \mathrm{~min}$. Reaction products were visualized by incubation with diaminobenzidine for $60 \mathrm{sec}$ at room temperature and then counterstained with hematoxylin for $5 \mathrm{~min}$ at room temperature. As a negative control, tissue samples were subject to the same staining procedures without incubation with primary antibodies. Regions of positive staining were quantified by calculating the pixel density using analysis LS Research image analysis software v5.0 (Olympus Soft Imaging Solutions $\mathrm{GmbH}$, Münster, Germany).

Reverse transcription-quantitative polymerase chain reaction $(R T-q P C R)$. The mice were injected intradermally into each ear with $30 \mu \mathrm{l} \mathrm{PBS}$, either alone or with $3 \mu \mathrm{g}$ recombinant mouse IL-17A once a day for two consecutive days. Following 1 week, the ear tissues were collected and the expression of miR-203a-3p.1 in ear tissues was determined by RT-qPCR. Total RNA from ear tissues was extracted using TRIzol (Takara Biotechnology Co., Ltd., Dalian, China) following the manufacturer's instructions. Single strand cDNA was synthesized using the PrimeScript miRNA cDNA Synthesis kit (Takara Biotechnology Co., Ltd.) using $2 \mu \mathrm{g}$ of total RNA as a template according to the manufacturer's instructions. qPCR was performed using SYBR Premix Ex Taq (Takara Biotechnology Co., Ltd.) according to the manufacturer's instructions. The thermocycling conditions were as follows: $95^{\circ} \mathrm{C}$ for $30 \mathrm{sec}$, followed by 45 cycles of $95^{\circ} \mathrm{C}$ for $5 \mathrm{sec}$ and $53^{\circ} \mathrm{C}$ for $20 \mathrm{sec}$. The expression of U6 small nuclear B non-coding RNA was used as an internal normalization control. The primers used were as follows: miR-203a-3p.1 primer, 5'-TAC GAGTGAAATGTTTAGGACCACTAG-3'; U6, 5'-ATTGGA ACGATACAGAGAAGATT-3'.

HaCaT cells were treated with medium only or with IL-17 for $48 \mathrm{~h}$, in the presence or absence of miR-203 inhibitor for $24 \mathrm{~h}$. The expression of suppressor of cytokine signaling 3 (SOCS3) in HaCaT cells was then determined by RT-qPCR. cDNA was synthesized using the PrimeScript RT-PCR kit (Takara Biotechnology Co., Ltd.) using $2 \mu \mathrm{g}$ of total RNA as a template according to the manufacturer's instructions. qPCR was performed using SYBR Premix Ex Taq (Takara Biotechnology Co., Ltd.) according to the manufacturer's instructions. The following thermocycling conditions were used: $95^{\circ} \mathrm{C}$ for $30 \mathrm{sec}$, followed by 45 cycles of $95^{\circ} \mathrm{C}$ for $5 \mathrm{sec}$ and $60^{\circ} \mathrm{C}$ for $20 \mathrm{sec}$. $\beta$-actin was used to normalize the expression levels of SOCS3. The primers used were as follows: SOCS3 forward, 5'-TGGATGGAGCGGGAGGCT-3', and reverse, 5'-ACGGACATCTTTCACCTCAGGCTCCT-3'; $\beta$-actin forward, 5'-GACAGGATGCAGAAGGAGATTACT-3' and reverse, 5'-TGATCCACATCTGCTGGAAGGT-3'. All RT-qPCR experiments were performed in triplicate using the Applied Biosystems 7500 Real-Time PCR system (Applied Biosystems; Thermo Fisher Scientific, Inc., Waltham, MA, USA). All primers were synthesized by Guangzhou RiboBio Co., Ltd. (Guangzhou, China). Samples were analyzed in triplicate, and the mean quantification cycle $\left(\mathrm{C}_{\mathrm{q}}\right)$ was calculated. Gene expression levels were analyzed by comparing the $\Delta \mathrm{C}_{\mathrm{q}}$ values of samples [where $\Delta \mathrm{C}_{\mathrm{q}}=\mathrm{C}_{\mathrm{q} \text { (target gene) }} \mathrm{C}_{\mathrm{q} \text { (housekeeping gene) }}$ ] transformed to a linear scale $\left(2^{-\Delta \Delta \mathrm{Cq}}\right)(20)$.

Cell culture. The human umbilical vein endothelial cells (HUVECs) cell line was purchased from the American Type Culture Collection (ATTC; Manassas, VA, USA). Cells were cultured in RPMI-1640 medium supplemented with $10 \%$ fetal bovine serum (both from Gibco; Thermo Fisher Scientific, Inc.), $100 \mathrm{U} / \mathrm{ml}$ penicillin and $100 \mu \mathrm{g} / \mathrm{ml}$ streptomycin, and maintained in a humidified atmosphere of $5 \% \mathrm{CO}_{2}$ at $37^{\circ} \mathrm{C}$. The human keratinocyte cell lines, HaCaT and HEK293T (both from ATCC), were incubated in Dulbecco's modified Eagle's medium containing 10\% fetal bovine serum (both from Gibco; Thermo Fisher Scientific, Inc.), $100 \mathrm{U} / \mathrm{ml}$ penicillin and $100 \mu \mathrm{g} / \mathrm{ml}$ streptomycin, and maintained in a humidified atmosphere of $5 \% \mathrm{CO}_{2}$ at $37^{\circ} \mathrm{C}$.

Cell transfection. HaCaT cells were seeded in 6-well plates ( $2 \times 10^{5} /$ well) for $24 \mathrm{~h}$ prior to transfection. Lipofectamine 2000 (Invitrogen; Thermo Fisher Scientific, Inc.) was used to transfect $\mathrm{HaCaT}$ cells with $50 \mathrm{nmol}$ miR-203 mimic, $150 \mathrm{nmol}$ miR-203 inhibitor or the same concentration of their respective negative controls (Guangzhou RiboBio Co., Ltd.), according to the manufacturer's instructions. The sequence of miR-203 mimic, miR-203 inhibitor and their respective negative controls were as follows: miR-203a-3p.1 mimic, 5'-GUGAAAUGUUUAGGACCACUAG-3' and 5'-AGUGGUCCUAAACAUUUCACUU-3'; miR-203a-3p.1 mimic control, 5'-UUCUCCCAACGUGUCACGUTT-3' and 5'-ACGUGACACGUUCGGAGAATT-3'; miR-203a-3p.1 
inhibitor, 5'-CUAGUG GUCCUA AACAUU UCAC-3'; miR-203a-3p.1 inhibitor control, 5'-CAGUACUUUUGU GUAGUACAA-3'. HaCaT cells were co-transfected with $150 \mathrm{nmol}$ SOCS3 small interfering (si)RNA or scrambled siRNA (Shanghai GenePharma Co., Ltd., Shanghai, China) and $150 \mathrm{nmol} \mathrm{miR-203}$ inhibitor using Lipofectamine 2000 according to the manufacturer's instructions (Invitrogen; Thermo Fisher Scientific, Inc.). At $24 \mathrm{~h}$ following transfection, $\mathrm{HaCaT}$ cells were treated with $80 \mathrm{ng} / \mathrm{ml}$ IL-17 (PeproTech, Inc., Rocky Hill, NJ, USA) for $48 \mathrm{~h}$ at $37^{\circ} \mathrm{C}$, then cells $\left(\sim 1.5 \times 10^{6} /\right.$ well $)$ were harvested for analysis. HUVECs were treated with conditioned $\mathrm{HaCaT}$ cell medium only at $37^{\circ} \mathrm{C}$ for $7 \mathrm{~h}$.

Luciferase reporter assays. For luciferase activity analysis, the 3'-UTR sequence of SOCS3 or the mutant SOCS3 3'-UTR sequence, which included a mutation in the miR-203 binding site, was cloned into a pGL3-promoter vector (Promega Corporation, Madison, WI, USA). Then HEK293T cells were co-transfected with $500 \mathrm{ng}$ wild-type pGL3-SOCS3-3'UTR or mutant pGL3-SOCS3-3'UTR and 10 pmol miR-203 mimic or 10 pmol miRNA control using Lipofectamine 2000, according to the manufacturer's instructions (Invitrogen; Thermo Fisher Scientific, Inc.). At $24 \mathrm{~h}$ following transfection, luciferase activity was detected using a dual-luciferase reporter assay system according to the manufacturer's instructions (Promega Corporation). Luciferase activity was normalized to Renilla luciferase activity.

Computational prediction. The target gene of miR-203 was predicted using TargetScan Release 7.1 software (www.targetscan.org/vert_71/).

Western blot analysis. HaCaT cells treated with medium alone or with IL-17 for $48 \mathrm{~h}$, in the presence or absence of miR-203 inhibitor for $24 \mathrm{~h}$. Total protein was extracted using a protein extraction kit (Beyotime Institute of Biotechnology, Haimen, China) according to the manufacturer's protocol. Protein concentration was determined using a bicinchoninic protein assay kit (Beyotime Institute of Biotechnology). For western blotting analysis, an equal quantity of total protein (30 $\mu \mathrm{g} / \mathrm{lane})$ was loaded, and separated by $8 \%$ SDS-PAGE. Following electrophoresis, proteins were transferred to a polyvinylidene difluoride membrane and blocked for $1 \mathrm{~h}$ at room temperature with 5\% milk in TBS with $0.1 \%$ Tween-20. The membrane was incubated with primary antibodies against the IL-17 receptor (cat no. ab180904; 1:1,000 dilution; Abcam), phosphorylated (p)-JAK2 (cat no. 4406; 1:1,000 dilution), p-STAT3 (cat no. 9145; 1:1,000 dilution), $\beta$-actin (cat no. 4970; 1:1,000 dilution), SOCS1 (cat no. 3950; 1:1,000 dilution) and SOCS3 (cat no. 2932; 1:1,000 dilution) (all from Cell Signaling Technology, Inc., Danvers, MA, USA) at $4^{\circ} \mathrm{C}$ overnight. The blots were subsequently incubated with a with horseradish peroxidase-conjugated secondary antibody (cat no. E030120-02; 1:2,000 dilution; EarthOx Life Sciences, Millbrae, CA, USA) for $1 \mathrm{~h}$ at room temperature. Proteins were visualized using Clarity Western ECL Substrate (Bio-Rad Laboratories, Inc., Hercules, CA, USA) according to the manufacturer's protocol. Densitometry analysis of the western blots was achieved using ImageJ v1.48 software (National Institutes of Health, Bethesda, MD, USA).
Tube formation assay. The tube formation assay was performed as described previously (21). Briefly, a 96-well plate was coated with $60 \mu 1$ Matrigel (BD Biosciences, Franklin Lakes, NJ, USA), which was allowed to polymerize and solidify at $37^{\circ} \mathrm{C}$ for $30 \mathrm{~min}$. HUVECs $\left(2 \times 10^{4}\right.$ cells $)$ were seeded onto the Matrigel layer in the presence or absence of conditioned medium from HaCaT cells treated with IL-17 $(80 \mathrm{ng} / \mathrm{ml}$ for $48 \mathrm{~h}$ ), miR-203 inhibitor ( $150 \mathrm{nmol}$ for $24 \mathrm{~h}$ ) or inhibitor control $(150 \mathrm{nmol}$ for $24 \mathrm{~h}$ ), and SOCS3-siRNA (150 $\mathrm{nmol}$ for $24 \mathrm{~h}$ ) or siRNA-control (150 nmol for $24 \mathrm{~h}$ ), and cells were incubated at $37^{\circ} \mathrm{C}$ for $7 \mathrm{~h}$. The number of blood-vessel-like tubules from six fields of view selected at random were counted, and images were captured using an inverted light microscope (Nikon Corporation, Tokyo, Japan).

Enzyme-linked immunosorbent assay (ELISA). HaCaT cells were treated with IL-17 ( $80 \mathrm{ng} / \mathrm{ml}$ for $48 \mathrm{~h}$ ), miR-203 inhibitor $(150 \mathrm{nmol}$ for $24 \mathrm{~h})$ or inhibitor control (150 nmol for $24 \mathrm{~h}$ ), and SOCS3-siRNA (150 nmol for $24 \mathrm{~h}$ ) or siRNA-control $(150 \mathrm{nmol}$ for $24 \mathrm{~h})$. The cell culture media were centrifuged at $1,500 \mathrm{x} \mathrm{g}$ for $10 \mathrm{~min}$ at $4^{\circ} \mathrm{C}$, the supernatants were collected and and stored at $-80^{\circ} \mathrm{C}$ prior to ELISA analysis. The level of VEGF secretion in $\mathrm{HaCaT}$ cell cultures were measured by an ELISA kit (cat no. DVE00; R\&D Systems, Minneapolis, MN, USA) according to the manufacturer's instructions.

Statistical analysis. Statistical analysis was performed using SPSS v13.0 software (SPSS, Inc., Chicago, IL, USA). The results were analyzed by one-way analysis of variance followed by a Student-Newman-Keuls post hoc test, and presented as the mean \pm standard deviation. $\mathrm{P}<0.05$ was considered to indicate a statistically significant difference. All experiments were repeated three times.

\section{Results}

miR-203 is upregulated in the ears of IL-17-stimulated mice and IL-17-treated HaCaT cells. In order to investigate the effect of miR-203 on IL-17-induced VEGF expression, miR-203, VEGF and CD34 expression in the ears of untreated and IL-17-stimulated mice were first examined. The results demonstrated that the expression of miR-203, VEGF and CD34 were significantly upregulated in the ears of IL-17-stimulated mice compared with the normal untreated group (Fig. 1A-C). The expression of miR-203 and VEGF secretion in IL-17-stimulated HaCaT cells was then investigated. Consistent with the in vivo results, miR-203 expression and VEGF secretion levels were significantly increased in HaCaT cells stimulated with IL-17 (Fig. 1D and E).

Suppression of miR-203 inhibits IL-17-induced VEGF secretion in HaCaT cells. To explore the role of miR-203 in IL-17-induced VEGF secretion, an miR-203 inhibitor was transfected into the HaCaT cells prior to stimulation with IL-17. As demonstrated in Fig. 2A, transfection of HaCaT cells with the miR-203 inhibitor was associated with a significant reduction in relative miR-203 expression levels when compared with cells transfected with the inhibitor control. IL-17-stimulation was associated with a significant increase in VEGF mRNA and the levels of VEGF in the supernatant of HaCaT cells, 
A

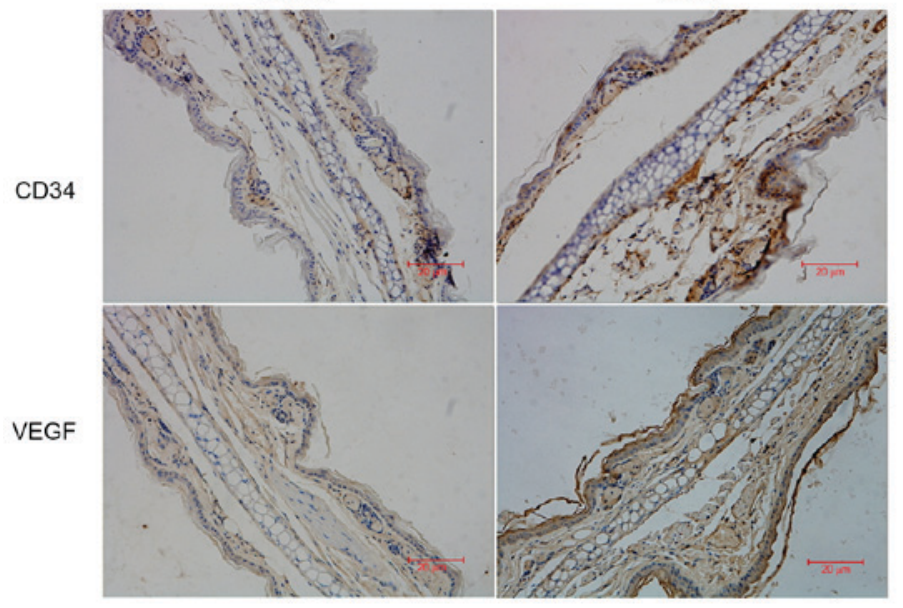

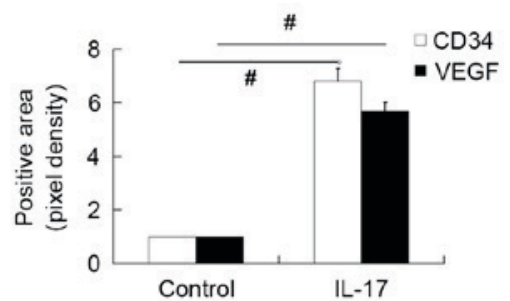

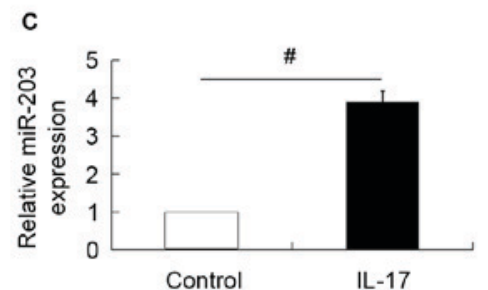

D

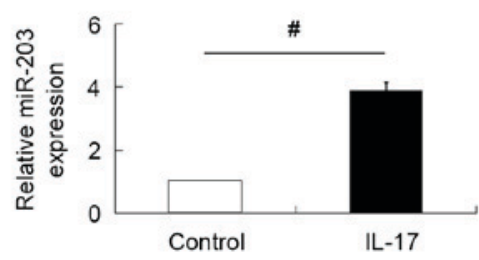

E

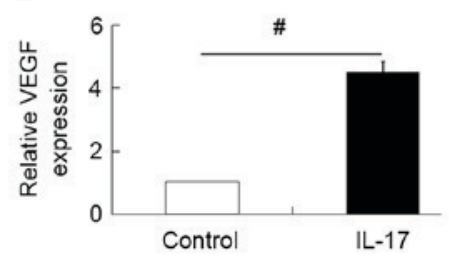

Figure 1. miR-203 is upregulated in the ears of IL-17-stimulated mice and IL-17-treated HaCaT cells. A total of $3 \mu \mathrm{g}$ recombinant mouse IL-17A was intradermally injected into the ears of mice for two consecutive days. (A) Ear tissues were collected at $24 \mathrm{~h}$ following the final administration of IL-17 and the expression of CD34 and VEGF in normal untreated mice and IL-17-stimulated mice was determined by immunohistochemistry (scale bar, $20 \mu \mathrm{m}$ ) and (B) quantified. (C) The relative expression of miR-203 in the ear tissues of normal untreated and IL-17-stimulated mice was determined by RT-qPCR. The results are presented as the mean \pm standard deviation ( $\mathrm{n}=8$ ). (D) HaCaT cells were treated with $80 \mathrm{ng} / \mathrm{ml} \mathrm{IL-17}$ for $48 \mathrm{~h}$, and the relative expression level of miR-203 in normal and IL-17-treated HaCaT cells was determined by RT-qPCR. The expression of miR-203 was normalized to that of U6. (E) VEGF secretion level in normal and IL-17-treated $\mathrm{HaCaT}$ cells was determined by enzyme-linked immunosorbent assay analysis. The results are presented as the mean \pm standard deviation $(n=3) .{ }^{~} \mathrm{P}<0.05$ as indicated. miR, microRNA; IL, interleukin; $\mathrm{CD}$, cluster of differentiation; VEGF, vascular endothelial growth factor; RT-qPCR, reverse transcription-quantitative polymerase chain reaction.

A

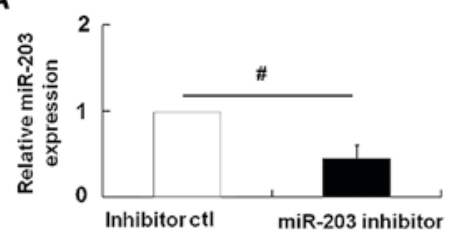

D

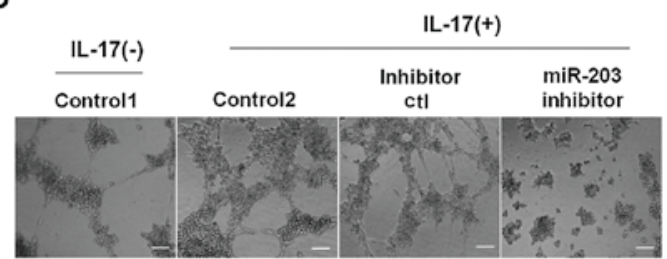

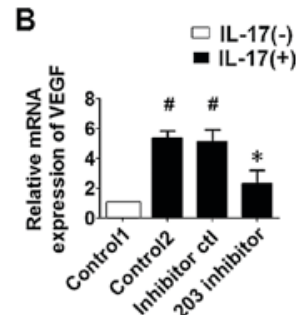

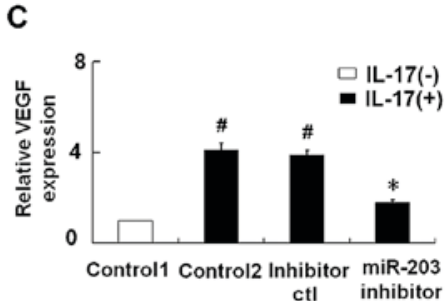

E

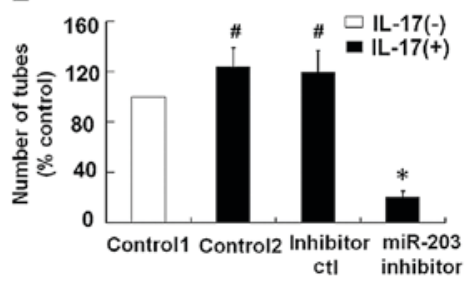

Figure 2. Suppression of miR-203 inhibits IL-17-induced VEGF secretion in HaCaT cells. Relative expression of (A) miR-203 and (B) VEGF mRNA in $\mathrm{HaCaT}$ cells transfected with an miR-203 inhibitor or an inhibitor ctl at $48 \mathrm{~h}$ following IL-17 stimulation, as determined by reverse transcription-quantitative polymerase chain reaction analysis. U6 and $\beta$-actin were used as endogenous controls for miR-203 and VEGF expression, respectively. (C) VEGF levels in the supernatant of $\mathrm{HaCaT}$ cells were determined using an enzyme-linked immunosorbent assay. HUVECs were seeded onto a Matrigel layer in the presence of conditioned medium from different groups of $\mathrm{HaCaT}$ cells for $7 \mathrm{~h}$. (D) Blood-vessel-like tubules from six fields of view selected at random were visualized under an inverted light microscope (scale bar, $100 \mu \mathrm{m}$ ) and (E) counted. The controll group consisted of untransfected HaCaT cells that were not treated with IL-17, whereas the control 2 group consisted of untransfected $\mathrm{HaCaT}$ cells that were treated with IL-17. The results are presented as the mean \pm standard deviation. All experiments were repeated three times. ${ }^{~} \mathrm{P}<0.05$ vs. control1; ${ }^{*} \mathrm{P}<0.05$ vs. control2. miR, microRNA; IL, interleukin; VEGF, vascular endothelial growth factor; ctl, control.

while repression of miR-203 significantly inhibited the IL-17-induced upregulation of VEGF levels (Fig. 2B and C). In addition, the effect of miR-203 on IL-17-induced VEGF secretion was assessed using a tube formation assay in HUVECs. 


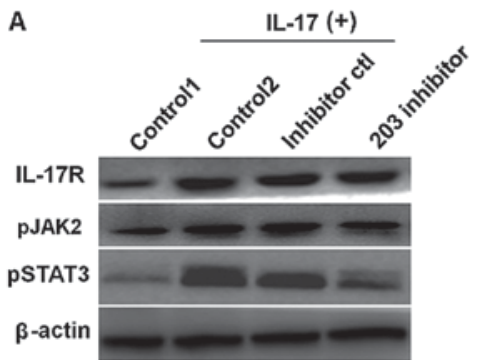

D

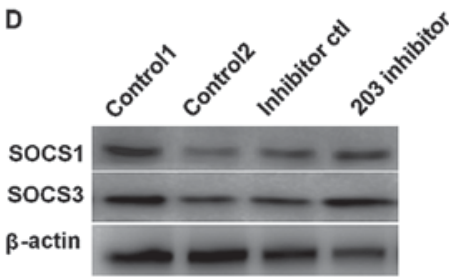

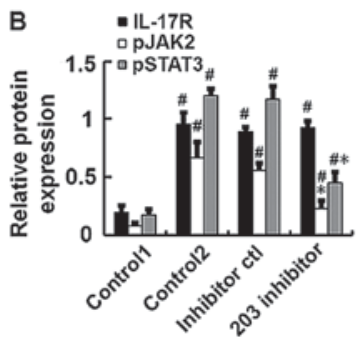
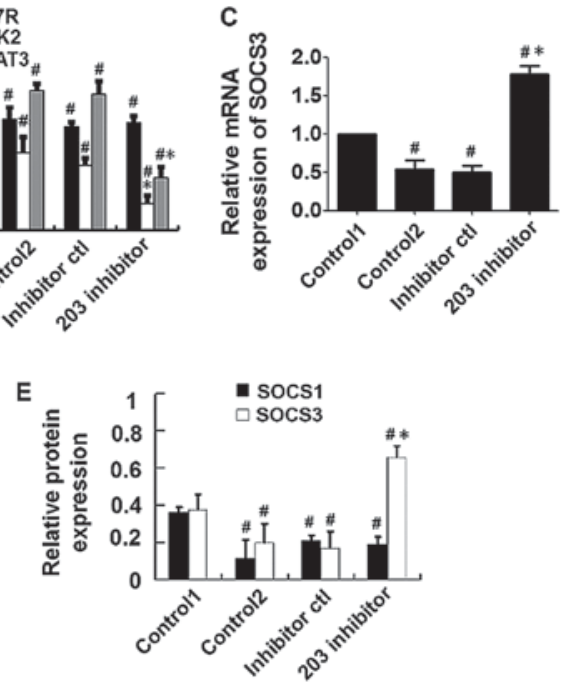

Figure 3. Repression of miR-203 inhibited IL-17-induced activation of the JAK2/STAT3 signaling pathway. HaCaT cells were transfected with miR-203 inhibitor for $24 \mathrm{~h}$ and subsequently treated with $80 \mathrm{ng} / \mathrm{ml} \mathrm{IL-17} \mathrm{for} \mathrm{a} \mathrm{further} 48 \mathrm{~h}$. (A) Western blot analysis of the protein expression levels of IL-17R, pJAK2 and pSTAT3, and (B) quantification of the results by densitometry analysis using Image J software. (C) The mRNA levels of SOCS3 were measured by reverse transcription-quantitative polymerase chain reaction analysis, and $\beta$-actin was used as an endogenous control. (D) Western blot analysis of the protein expression levels of SOCS1 and SOCS3 inhibitors of the JAK2/STAT3 signaling pathway, and (E) quantification of the results by densitometry analysis using ImageJ software. Target protein levels were normalized to $\beta$-actin. The control1 group consisted of untransfected HaCaT cells that were not treated with IL-17, whereas the control 2 group consisted of untransfected HaCaT cells that were treated with IL-17. The results are presented as the mean \pm standard deviation $(\mathrm{n}=3) .{ }^{\#} \mathrm{P}<0.05$ vs. control1; *P<0.05 vs. control2. miR, microRNA; IL, interleukin; JAK, Janus kinase; STAT, signal transducer and activator of transcription; SOCS, suppressor of cytokine signaling; p-, phosphorylated; R, receptor.

Consistent with the results observed in HaCaT cells, suppression of miR-203 significantly attenuated the IL-17-induced upregulation of VEGF levels and significantly inhibited tube formation of HUVECs (Fig. 2B-E).

Repression of miR-203 inhibits IL-17-induced activation of the JAK2/STAT3 signaling pathway. In order to explore the effect of miR-203 on IL-17-induced activation of the JAK2/STAT3 signaling pathway, HaCaT cells were transfected with miR-203 inhibitor or controls for $24 \mathrm{~h}$, then treated with $80 \mathrm{ng} / \mathrm{ml} \mathrm{IL-17}$ for a further $48 \mathrm{~h}$. Protein expression levels were subsequently detected by western blot analysis. The results demonstrated that the expression levels of p-JAK2 and p-STAT3 were significantly reduced in miR-203 inhibitor-transfected cells when compared with IL-17-stimulated and inhibitor control-transfected cells (Fig. 3A and B). The next aim was to identify targets of miR-203. As demonstrated in (Fig. 3C-E), the mRNA and protein expression levels of SOCS3, an inhibitor of the JAK2/STAT3 signaling pathway, was significantly upregulated in miR-203 inhibitor-transfected cells when compared with IL-17-stimulated and inhibitor control-transfected cells. By contrast, western blot analysis demonstrated that the protein expression levels of SOCS1, an additional inhibitor of the JAK2/STAT3 signaling pathway, were not significantly affected by miR-203 inhibition (Fig. 3D and E). These results suggest that miR-203 may inhibit JAK2/STAT3 signaling via targeting of SOCS3 expression.

miR-203 directly targets SOCS3. To assess the role of miR-203 in regulating the JAK2/STAT3 signaling pathway, computational analysis (TargetScan v7.1) was performed to predict the potential target genes of miR-203. The results indicated that miR-203 may target sequences in 3'-untranslated region (UTR) of SOCS3 (Fig. 4A). The 3'-UTR sequence of SOCS3 was then cloned into a luciferase reporter plasmid. A mutant SOCS3 3'-UTR sequence, which included a mutation in the miR-203 binding site, was additionally cloned into the luciferase reporter plasmid (Fig. 4A). The dual-luciferase reporter assay was performed using HEK293T cells. The results demonstrated that the luciferase activity in HEK293T cells co-transfected with an miR-203 mimic and a luciferase reporter plasmid containing the wild type SOCS3 3'-UTR sequence was significantly reduced when compared with the mimic control (Fig. 4B). By contest, no significant alterations in luciferase activity were observed HEK293T cells transfected with miR-203 mimic and a luciferase reporter plasmid containing the mutant SOCS3 3'-UTR sequence (Fig. 4B). In addition, transfection of $\mathrm{HaCaT}$ cells with miR-203 mimics was associated with a significant reduction in the mRNA and protein expression levels of SOCS3 (Fig. 4C-E). These results provide evidence to suggest that SOCS3 may be a direct target of miR-203.

Repression of miR-203 attenuates IL-17-induced VEGF secretion in HaCaT cells via targeting SOCS3. To further confirm the role of SOCS3 in miR-203-mediated VEGF secretion in response to IL-17 stimulation, HaCaT cells were co-transfected with an miR-203 inhibitor together with an siRNA targeting SOCS3, and then stimulated with IL-17. The results demonstrated that treatment of cells with the SOCS3 siRNA significantly attenuated the effects of the miR-203 inhibitor on the IL-17-induced upregulation of VEGF levels and increase in tube formation (Fig. 5). These results suggest that IL-17 may induce miR-203 expression, which may subsequently increase VEGF secretion via targeting of SOCS3. 
A

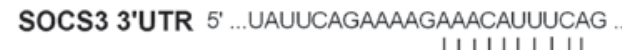

|| ||||||||

Hsa-miR-203 3' - GAUCACCAGGAUUUGUAAAGUG -5'

Mutant SOCS3 3'UTR 5' ... UAUUCAGAAAAGAGAUACUGUAG ...
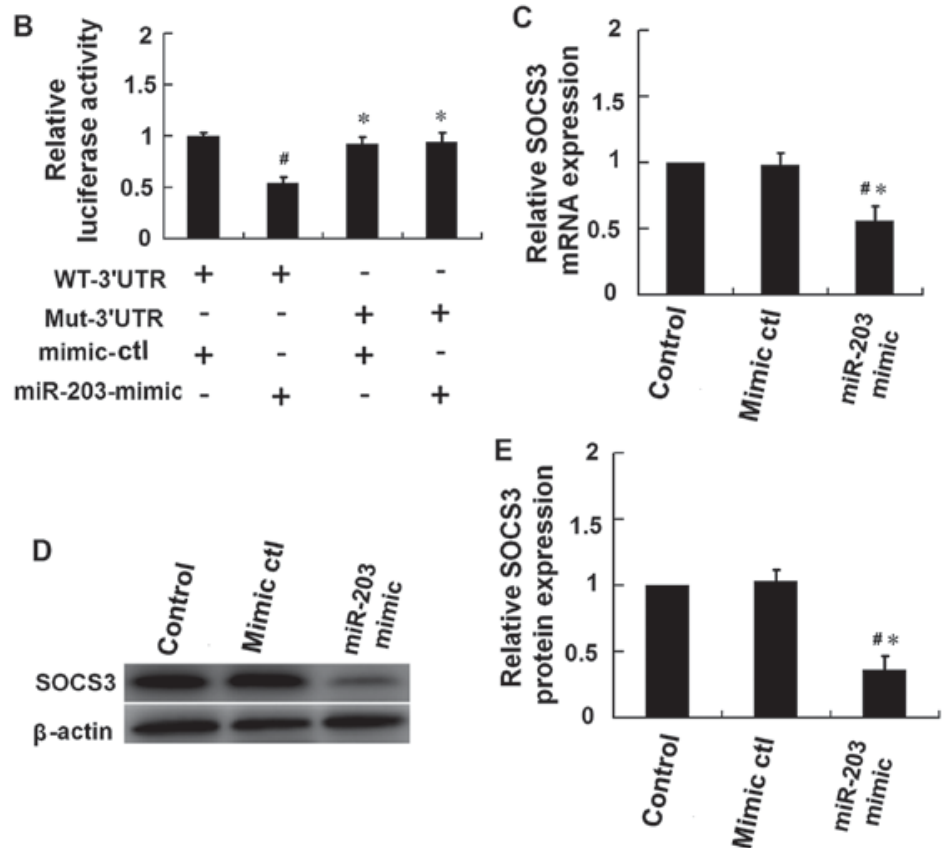

Figure 4. SOCS3 is the molecular target of miR-203. (A) Schematic of the predicted miR-203 binding site in the 3'-UTR region of SOCS3, as detected using TargetScan v7.1 software. The mutant SOCS3 3'-UTR sequence included several mutations in the miR-203 binding site. (B) A dual-luciferase reporter assay was performed using HEK293T cells. Cells were co-transfected with a reporter vector containing the WT-3'UTR or the Mut-3'UTR, in addition to a miR-203-mimic or a mimic-ctl. The firefly/Renilla activity ratio was calculated to determine the luciferase activity $\left({ }^{*} \mathrm{P}<0.05\right.$ vs. HaCaT cells transfected with WT-3'UTR plus mimic-ctl; " $\mathrm{P}<0.05$ vs. HaCaT cells transfected with WT-3-UTR plus miR-203 mimic). Following 48 h of IL-17 stimulation, HaCaT cells were then transfected with an miR-203 mimic or mimic-ctl, and cells were harvested at $24 \mathrm{~h}$ following transfection. The (C) mRNA and (D) protein levels of SOCS3 were measured by western blotting and reverse transcription-quantitative polymerase chain reaction analysis, respectively, and (E) densitometry analysis of the western blots was performed using ImageJ software. SOCS3 mRNA and protein expression levels were normalized to that of $\beta$-actin $\left({ }^{\#} \mathrm{P}<0.05 \mathrm{vs}\right.$. control; ${ }^{*} \mathrm{P}<0.05$ vs. mimic-ctl). The results are presented as the mean \pm standard deviation $(\mathrm{n}=3)$. SOCS, suppressor of cytokine signaling; miR, microRNA; UTR, untranslated region; WT-3'UTR, pGL3 vector containing the wild-type SOCS3 3'-UTR; Mut-3'UTR, pGL3 vector containing the mutant SOCS3 3'-UTR; ctl, control.

A

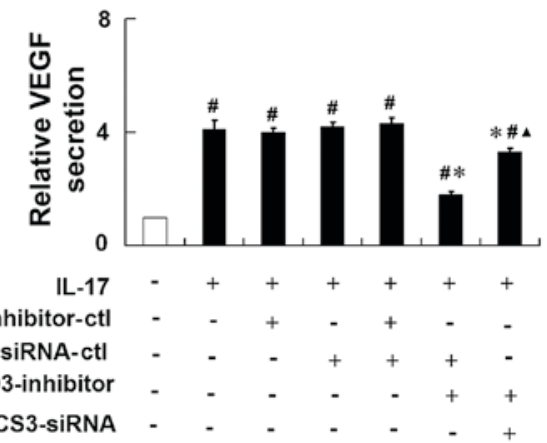

C IL-17(-)

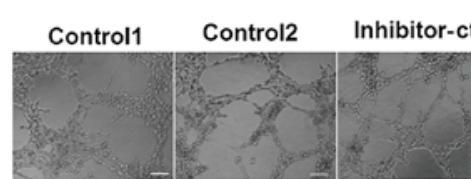

B

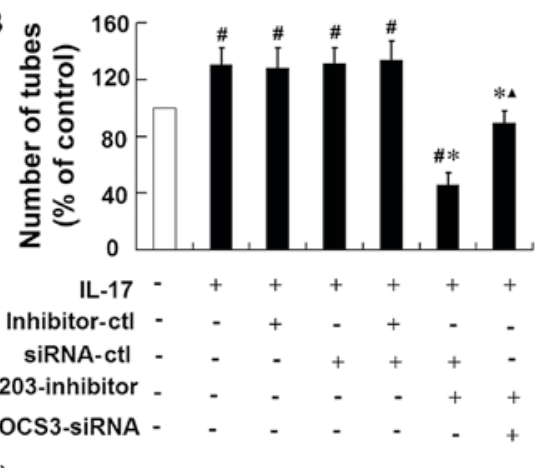

$\mathrm{IL}-17(+)$ 


\section{Discussion}

The results of the present study demonstrated that miR-203 expression is significantly upregulated in mice and $\mathrm{HaCaT}$ cells stimulated with IL-17. In addition, VEGF levels were observed to increase in the ears of IL-17-stimulated mice and in the supernatant of IL-17-treated HaCaT cells. These results suggest that IL-17 may induce VEGF expression, and that miR-203 may be involved in mediating this effect. In addition, the results indicated that inhibition of miR-203 reversed the IL-17-induced increase in VEGF secretion and inhibited the IL-17-induced activation of JAK2/STAT3 signaling. Furthermore, the present study provided evidence to suggest that SOCS3, a repressor of the JAK2/STAT3 signaling pathway, may be a direct target of miR-203.

miRNAs are a class of abundant non-coding RNA molecules that modulate mRNA degradation or inhibition of translation by specifically binding to the 3'-UTR of target mRNA sequences (22-24). miRNAs serve key roles in the regulation of cell differentiation, growth and death in normal and malignant tissues $(25,26)$. miR-203 is a miRNA preferentially expressed in the skin, and is an important regulator of keratinocyte differentiation (18). miR-203 has been implicated in a number of skin diseases, particularly psoriasis, via regulation of pro-inflammatory cytokines $(27,28)$. However, a complete understanding of the mechanisms underlying the involvement of miR-203 in psoriasis is lacking. The results of the present study demonstrated that miR-203 expression is significantly upregulated in mice and $\mathrm{HaCaT}$ cells stimulated with IL-17. In addition, VEGF levels were increased in the ears of IL-17-stimulated mice and in the supernatant of IL-17-treated HaCaT cells. These results implied that miR-203 may be involved in IL-17-induced VEGF expression. In addition, the results of the present study demonstrated that miR-203 may function as a positive effector of IL-17-induced VEGF secretion, as inhibition of miR-203 effectively reversed IL-17-induced VEGF secretion.

Further investigation of the mechanisms by which miR-203 functions to mediate the IL-17-induced increase in VEGF secretion demonstrated that SOCS3 may be a direct target of miR-203 via binding to its 3'-UTR, thus inhibiting SOCS3 expression. An inverse association between miR-203 and SOCS3 expression in HaCaT cells stimulated by IL-17 was observed. These results suggest that SOCS3 may be a direct target of miR-203, and potentially mediate the process of IL-17-induced VEGF expression in HaCaT cells.

In order to prevent the adverse effects of over-activation, the duration and intensity of JAK/STAT signaling pathway activation is strictly controlled by negative regulators (29). SOCS proteins are induced by growth factors or cytokines, and regulate the duration and magnitude of inflammatory responses activated by these cytokines via the inhibition of JAK proteins in a negative feedback loop (30). Previous studies have demonstrated that SOCS1 and SOCS3 proteins are the major regulators of the JAK2/STAT3 signaling pathway (31-33). The results of the present study demonstrated that SOCS1 and SOCS3 expression was significantly downregulated in $\mathrm{HaCaT}$ cells following stimulation with IL-17. However, only SOCS3 protein expression was upregulated following inhibition of miR-203 in IL-17-treated HaCaT cells. This provides additional evidence to suggest that SOCS3 may be a target of miR-203.

Angiogenesis is an important pathological feature of psoriasis, and the expression of a number of angiogenic mediators, including VEGF, increase during the development of psoriasis $(34,35)$. The present study demonstrated that IL-17 induces the expression of VEGF in vitro and in vivo, which is consistent with the results of previous studies $(13,15)$. Notably, suppression of miR-203 reversed IL-17-induced VEGF expression in $\mathrm{HaCaT}$ cells. These results are consistent with the observed upregulation of SOCS3 expression and the reduction in JAK/STAT signalling pathway activation in the IL-17 signaling process following inhibition of miR-203, which indicated that miR-203 may be involved in mediating IL-17-induced VEGF expression.

In conclusion, the results of the current study suggest that miR-203 may inhibit the expression of SOCS3 by directly binding to the 3'-UTR of SOCS3 and promoting the degradation of the SOCS3 mRNA, thus activating the JAK2/STAT3 signaling pathway and mediating IL-17-induced VEGF secretion. Future studies that aim to investigate the role of miR-203 further, may provide novel insights into its mechanisms of action, as well as identify potential therapeutic strategies for the treatment of psoriasis.

\section{Acknowledgements}

The present study was supported by the National Natural Science Foundation of China (grant nos. 81673055 and 81402595) and the Program for Liaoning Excellent Talents in University (grant no. LR2012026).

\section{References}

1. Schön MP and Boehncke WH: Psoriasis. N Eng1 J Med 352: 1899-1912, 2005

2. Wei T, Xu N, Meisgen F, Ståhle M, Sonkoly E and Pivarcsi A: Interleukin- 8 is regulated by miR-203 at the posttranscriptional level in primary human keratinocytes. Eur J Dermatol 19 , 2013.

3. Wolk K, Witte E, Wallace E, Döcke WD, Kunz S, Asadullah K, Volk HD, Sterry W and Sabat R: IL-22 regulates the expression of genes responsible for antimicrobial defense, cellular differentiation, and mobility in keratinocytes: A potential role in psoriasis. Eur J Immunol 36: 1309-1323, 2006.

4. Heidenreich R, Röcken M and Ghoreschi K: Angiogenesis: The new potential target for the therapy of psoriasis?. Drug News Perspect 21: 97-105, 2008.

5. Hongqin T, Xinyu L, Heng G, Lanfang X, Yongfang W and Shasha S: Triptolide inhibits IFN- $\gamma$ signaling via the Jak/STAT pathway in HaCaT keratinocytes. Phytother Res 25: 1678-1685, 2011.

6. Xu Y, Xu X, Gao X, Chen H and Geng L: Shikonin suppresses IL-17-induced VEGF expression via blockage of JAK2/STAT3 pathway. Int Immunopharmacol 19: 327-333, 2014.

7. Koga C, Kabashima K, Shiraishi N, Kobayashi M and Tokura Y: Possible pathogenic role of Th17 cells for atopic dermatitis. J Invest Dermatol 128: 2625-2630, 2008.

8. Primo MN, Bak RO, Schibler B and Mikkelsen JG: Regulation of pro-inflammatory cytokines TNF $\alpha$ and IL24 by microRNA-203 in primary keratinocytes. Cytokine 60: 741-748, 2012.

9. Cai Y, Fleming $C$ and Yan J: New insights of T cells in the pathogenesis of psoriasis. Cell Mol Immunol 9: 302-309, 2012.

10. Nickoloff BJ and Wrone-Smith T: Animal models of psoriasis. Nat Med 3: 475-476, 1997.

11. Zamore PD and Haley B: Ribo-gnome: The big world of small RNAs. Science 309: 1519-1524, 2005. 
12. Lerman G, Avivi C,Mardoukh C, Barzilai A, Tessone A, Gradus B, Pavlotsky F, Barshack I, Polak-Charcon S, Orenstein A, et al: MiRNA expression in psoriatic skin: Reciprocal regulation of hsa-miR-99a and IGF-1R. PLoS One 6: e20916, 2011.

13. Pittelkow MR: Psoriasis: More than skin deep. Nat Med 11: 17-18, 2005.

14. Chen CZ, Li L, Lodish HF and Bartel DP: MicroRNAs modulate hematopoietic lineage differentiation. Science 303: 83-86, 2004.

15. Creamer D, Sullivan D, Bicknell R and Barker J: Angiogenesis in psoriasis. Angiogenesis 5: 231-236, 2002.

16. Huh JE, Baek YH, Lee MH, Choi DY, Park DS and Lee JD: Bee venom inhibits tumor angiogenesis and metastasis by inhibiting tyrosine phosphorylation of VEGFR-2 in LLC-tumor-bearing mice. Cancer Lett 292: 98-110, 2010.

17. Cheng AM, Byrom MW, Shelton J and Ford LP: Antisense inhibition of human miRNAs and indications for an involvement of miRNA in cell growth and apoptosis. Nucleic Acids Res 33: 1290-1297, 2005.

18. Sonkoly E, Ståhle M and Pivarcsi A: MicroRNAs: Novel regulators in skin inflammation. Clin Exp Dermatol 33: 312-315, 2008

19. Cho A and Seok SH: Ethical guidelines for use of experimental animals in biomedical research. J Bacteriol Virol 43: 18-26, 2013

20. Livak KJ and Schmittgen TD: Analysis of relative gene expression data using real-time quantitative PCR and the 2(-Delta Delta C(T)) method. Methods 25: 402-408, 2001.

21. Xiong H, Du W, Zhang YJ, Hong J, Su WY, Tang JT, Wang YC, Lu R and Fang JY: Trichostatin A, a histone deacetylase inhibitor, suppresses JAK2/STAT3 signaling via inducing the promoter-associated histone acetylation of SOCS1 and SOCS3 in human colorectal cancer cells. Mol Carcinog 51: 174-184, 2012.

22. Bartel DP: MicroRNAs: Genomics, biogenesis, mechanism and function. Cell 116: 281-297, 2004.

23. Hueber W, Patel DD, Dryja T, Wright AM, Koroleva I, Bruin G, Antoni C, Draelos Z, Gold MH; Psoriasis Study Group, et al: Effects of AIN457, a fully human antibody to interleukin-17A, on psoriasis, rheumatoid arthritis and uveitis. Sci Transl Med 2: 52ra72, 2010

24. Zibert JR, Løvendorf MB, Litman T, Olsen J, Kaczkowski B and Skov L: MicroRNAs and potential target interactions in psoriasis. J Dermatol Sci 58: 177-185, 2010.
25. Darnell JE Jr: STATs and gene regulation. Science 277: $1630-1635,1997$.

26. Valencia-Sanchez MA, Liu J, Hannon GJ and Parker R: Control of translation and mRNA degradation by miRNAs and siRNAs. Genes Dev 20: 515-524, 2006.

27. Starnes T, Robertson MJ, Sledge G, Kelich S, Nakshatri H, Broxmeyer HE and Hromas R: Cutting edge: IL-17F, a novel cytokine selectively expressed in activated T cells and monocytes, regulates angiogenesis and endothelial cell cytokine production. J Immunol 167: 4137-4140, 2001.

28. Zhang Y, Zhou B, Zhang F, Wu J, Hu Y, Liu Y and Zhai Q: Amyloid- $\beta$ induces hepatic insulin resistance by activating JAK2/STAT3/SOCS-1 signaling pathway. Diabetes 61: 1434-1443, 2012.

29. Nickoloff BJ: Cracking the cytokine code in psoriasis. Nat Med 13: 242-244, 2007.

30. Song B, Jin H, Yu X, Zhang Z, Yu H, Ye J, Xu Y, Zhou T, Oudit GY, Ye JY, et al: Angiotensin-converting enzyme 2 attenuates oxidative stress and VSMC proliferation via the JAK2/STAT3/SOCS3 and profilin-1/MAPK signaling pathways. Regul Pept 185: 44-51, 2013.

31. Zheng Y, Danilenko DM, Valdez P, Kasman I, Eastham-Anderson $\mathrm{J}$, Wu J and Ouyang W: Interleukin-22, a $\mathrm{T}(\mathrm{H}) 17$ cytokine, mediates IL-23-induced dermal inflammation and acanthosis. Nature 445: 648-651, 2007.

32. Krebs DL and Hilton DJ: SOCS: Physiological suppressors of cytokine signaling. J Cell Sci 113: 2813-2819, 2000.

33. Numasaki M, Fukushi J, Ono M, Narula SK, Zavodny PJ, Kudo T, Robbins PD, Tahara $\mathrm{H}$ and Lotze MT: Interleukin-17 promotes angiogenesis and tumor growth. Blood 101: 2620-2627, 2003.

34. Liew SC, Das-Gupta E, Chakravarthi S, Wong SF, Lee N, Safdar N and Jamil A: Differential expression of the angiogenesis growth factors in psoriasis vulgaris. BMC Res Notes 5: 201, 2012.

35. Sankar L, Arumugam D, Boj S and Pradeep P: Expression of angiogenic factors in psoriasis vulgaris. J Clin Diagn Res 11: EC23-EC27, 2017. 